Syphilis

\section{Congenital syphilis in the United Kingdom}

\section{Simms, H Ward}

\section{Are we prepared?}

W orldwide, the World Health Organization estimates that maternal syphilis is responsible for 460000 stillbirths or abortions, 270000 cases of congenital syphilis, and 270000 low birthweight or premature babies. ${ }^{1}$ The vast majority of these cases are seen in developing countries, but congenital syphilis also occurs in affluent nations. Established control methods are based on antenatal screening in the first trimester supported by treatment and partner notification. High risk patients are then followed up in the third trimester; treatment and partner notification are undertaken where necessary. These control methods are highly cost effective but are dependent on well structured healthcare pathways. $^{23}$ A recent paper by Cross et al provides insight into the problems of managing syphilis in pregnancy across diverse clinical settings in a London teaching hospital. ${ }^{4}$ The authors report outcomes for 70 women with positive syphilis serology, and found that of 42 women at intermediate risk of transmitting infectious syphilis to their child, 26 $(62 \%)$ received no follow up serology. ${ }^{4}$ It is clear from this paper that secure procedures were not in place to ensure that women were appropriately referred, investigated, and managed.

The re-emergence of infectious syphilis (primary, secondary, and early latent) in the United Kingdom in the late 1990s was characterised by a series of outbreaks and foci. ${ }^{5}$ Although the majority of cases have been seen in men who have sex with men (MSM), cases reported among heterosexual men and women have been increasing. Between 2000 and 2004, diagnoses of syphilis rose by $583 \%$ (from 134 to 915 ) in heterosexual men and by $255 \%$ (from 78 to 277) in women. As disease incidence has risen cases of congenital syphilis have emerged, although there is no systematic national surveillance of these cases. Two case reports have been published. The first, from 2000, was in the context of a heterosexual outbreak in Peterborough ${ }^{5}$ and the second was reported in Walsall in $2002 .{ }^{6}$

Current UK guidelines suggest that all pregnant women should be tested in the first trimester. ${ }^{7}$ For those patients in whom infection is identified testing in the third trimester is also recommended, as are those who are thought to be at increased risk of infection. Both the recent published case studies of congenital syphilis were identified in the third trimester and one case had not attended for antenatal care. The identification and management of infection in women who are at high risk of infection yet who are marginalised in society is challenging, as they are unlikely to come into contact with healthcare services until delivery.

\section{Congenital syphilis is a preventable disease and its re-emergence in the United Kingdom reflects a failure of prenatal care delivery systems, as well as syphilis control programmes}

The number of cases of congenital syphilis that have occurred in the United Kingdom is unclear as there is no definitive surveillance tool. Surveillance of antenatal infections, including syphilis, has been undertaken in London since 2000. These data showed that the proportion of pregnant women screened at the 30 London maternity units rose from $89 \%$ in 2000 to $94 \%$ in 2002 . In 2002, 412 pregnant women (4/1000) had a positive test for syphilis. However, the IgG screening test is unable to distinguish between treated or untreated infection acquired either during or before pregnancy, and does not differentiate between infectious syphilis and other treponemal diseases such as yaws, which is largely an infection of childhood found in tropical countries.

The number of diagnosed cases of congenital syphilis is difficult to estimate. Diagnoses are recorded in the KC60 return from genitourinary medicine clinics but these are likely to be incomplete as cases are managed in a variety of clinical settings. Between 1994 and 1997, enhanced surveillance of congenital syphilis was undertaken by the Public Health Laboratory Service (now the Health Protection Agency) in collaboration with specialists in genitourinary medicine (the British Cooperative Clinical Group) and the British Paediatric Surveillance Unit (Royal College of Paediatrics and Child Health). ${ }^{8}$ Nine presumed cases of congenital syphilis were identified over the 3 years, this at the nadir of syphilis diagnosis in the United Kingdom. To estimate the burden of cases associated with congenital syphilis, this survey needs to be repeated and include specialists in obstetrics and gynaecology.

Congenital syphilis is a preventable disease and its re-emergence in the United Kingdom reflects a failure of prenatal care delivery systems, as well as syphilis control programmes. The investigation by Cross et al raises concerns about the effectiveness of the present control strategy. Anecdotal reports suggest that many more cases of congenital syphilis have been seen than have been reported in the literature, and if control strategies are not improved an increasing number of cases will emerge as the epidemic progresses.

Sex Transm Infect 2006;82:1. doi: $10.1136 /$ sti.2005.019349

\section{Authors' affiliations}

I Simms, H Ward, Health Protection Agency Centre for Infections, 61 Colindale Avenue, London NW9 5EQ, UK

Correspondence to: Dr lan Simms, Health Protection Agency Centre for Infections, 61 Colindale Avenue, London NW9 5EQ, UK. ian.simms@hpa.org.uk

\section{REFERENCES}

1 Finelli L, Berman SM, Koumans EH, et al. Congenital syphilis. Bull World Health Organ 1998;76:126-8.

2 Williams K. Screening for syphilis in pregnancy: an assessment of the costs and benefits. Commun Med 1985;7:35-42.

3 Goh BT. Syphilis in adults. Sex Transm Infect 2005:81:448-52.

4 Cross A, Luck S, Patey R, et al. Syphilis in London circa 2004: new challenges from an old disease. Arch Dis Childhood 2005;90:1045-6.

5 Simms I, Fenton KA, Ashton $M$, et al. The reemergence of syphilis in the UK: the new epidemic phases. Sex Transm Dis 2005:32:220-6.

6 Pugh RN, Laverty S, Simms I, et al. Syphilis outbreak in Walsall: case profiles and public health implications. Comm Dis Public Health 2004;7:27-9.

7 CDSC. Screening pregnant women for syphilis. Commun Dis Rep Wkly 2000; 10:367-70.

8 Hurtig A-K, Nicoll A, Carne C, et al. Syphilis in pregnant women and their children in the United Kingdom: results from national clinician reporting surveys 1994-7. BMJ 1998;317:1617-19. 